\title{
Tagungsbericht „Arbeit und Altern 2019“
}

G. Richter, L. Hünefeld, C. Kleinschmidt, J. Michel, M. Niehaus, M. Ribbat, M. Schmeink und C. Weber

Stand und Herausforderungen der alters- und alternsgerechten Arbeitsgestaltung

Im Jahr 1999 hatte eine internationale Tagung in Berlin den Wissensstand zu Altern und Arbeit zusammengetragen (Ergebnisse bei Rothkirch 2000). 20 Jahre danach haben nun Expertinnen und Experten aus Wissenschaft, Unternehmen, von Sozialpartner*innen und Sozialversicherungen den Stand des seither gewonnenen Wissens und der betrieblichen Umsetzung der Erkenntnisse bilanziert. Sie trafen sich bei der Tagung „Arbeit und Altern 2019 - Herausforderungen für Wirtschaft und Gesellschaft" in Dortmund, gemeinsam veranstaltet von der Bundesanstalt für Arbeitsschutz und Arbeitsmedizin (BAuA) und dem Unternehmensnetzwerk das Demographie Netzwerk e.V. (ddn). Das Ziel der Tagung: Den Erkenntnisfortschritt der vergangenen 20 Jahre diskursiv und generationenübergreifend aufzuarbeiten. Neben etablierten Expertinnen und Experten beteiligten sich auch Studentinnen und Studenten sowie Doktorandinnen und Doktoranden, sodass die Thematik nicht nur aus der Perspektive der Babyboomer-Generation beleuchtet wurde. Das umfassende Themenfeld der altersund alternsgerechten Arbeitsgestaltung wurde unter sieben Aspekten vertieft behandelt: Gesundheit und Krankheit; Weiterarbeiten im Rentenalter; soziale Ungleichheit und Gender; Lernen und Motivation; betriebliche und tarifliche Regelungen; Personalmanagement; Arbeitsgestaltung.

Eingangs skizzierten Anita Tisch und Götz Richter (beide BAuA) Ziele der Tagung und Anforderungen an eine umfassende Aufarbeitung des Themas. Im anschließenden Gespräch zwischen Jan Oltmanns (Mercedes Benz AG) und Gerhard Naegele (em. Prof. TU Dortmund) wurde deutlich, dass das "Defizitmodell des Alterns“ in den letzten 20 Jahren in Forschung und betrieblicher Praxis als überwunden angesehen werden kann. Das Klischee, dass Ältere automatisch an Leistungskraft verlieren oder nicht mehr lernfähig sind, ist in der Arbeitswelt weitgehend überwunden, erklärte Naegele. Dennoch wirke dieser „Defizitblick“ auf das Alter fort. Als eine Art Reflex der ökonomischen Überlegungen von Unternehmen: Wird es in den Firmenkassen eng, betrachtet man die Älteren schnell als 
verzichtbar. Werden die Älteren aber gebraucht, beispielsweise als Fachkräfte, wird eher positiv auf ihre Potenziale geschaut. Naegele wies darauf hin, dass die Heraufsetzung der Regelaltersgrenze nicht von Programmen oder Maßnahmen zur alters- und alternsgerechten Arbeitsgestaltung (insbesondere für kleine und mittlere Unternehmen) begleitet worden ist. Die Politik habe mit der Erhöhung der Regelaltersgrenze neue soziale Ungleichheiten erzeugt. Mit Blick auf anstehende wirtschaftliche Umbrüche müsse sich erst noch erweisen, ob nicht bereits überwunden geglaubte Instrumente wie der Vorruhestand in Zukunft wieder verstärkt genutzt werden. Jan Oltmanns, Personaler mit wissenschaftlichem Hintergrund, hatte auch die Mitmachausstellung „Ey Alter“ wissenschaftlich betreut. Sie hatte das Ziel, Altersstereotype aufzubrechen. Unter Verweis auf seine kürzlich fertiggestellte Dissertation zum Thema Plastizität erklärte er, dass Entwicklungspfade (positiv und negativ) veränderbar sind. Mit Blick auf den $\mathrm{Zu}$ sammenhang von Alterung und kognitiver Leistungsfähigkeit habe die Entwicklungspsychologie der Lebensspanne dazu beigetragen, den Blick weg von der Defizithypothese auf die Potenziale zu richten. Auch in einigen Unternehmen, so bei der Mercedes Benz AG, spielten Vorurteile von Jung gegenüber Alt oder umgekehrt im Moment keine große Rolle.

Lutz Bellmann (IAB) stellte schließlich den Erkenntnistand zu Erwerbstätigkeit, -minderung und -unfähigkeit sowie zu Pfaden in die Rente da. Die Erwerbsbeteiligung Älterer ist im Zeitraum 1995 von 2018 deutlich gestiegen. Das liegt vor allem daran, dass Ältere immer länger im Betrieb verbleiben. Die Suche nach einem neuen Arbeitsplatz gestaltet sich für Ältere dagegen schwer, insbesondere für ältere Arbeitslose. Die berufliche Neubzw. Umorientierung wird in der modernen Arbeitswelt jedoch immer wichtiger. Deshalb gewinnt die Weiterbildung neben der Erstausbildung zunehmend an Bedeutung. Die Weiterbildungsbeteiligung insgesamt ist im Zeitverlauf gestiegen. Dies gilt aber nicht für betriebliche Maßnahmen zur Erhaltung der Arbeits- und Beschäftigungsfähigkeit der Älteren. Insbesondere Betriebe mit weniger als 100 Beschäftigten halten sich dabei stark zurück.

1. Gesundheit und Krankheit. Den Stand des Wissens und offene Forschungsfragen zum Zusammenhang von Gesundheit und Krankheit mit Alter und Altern in der Arbeitswelt stellten Hermann Burr (BAuA) und Melanie Ebener (Universität Wuppertal) dar. Silvia Linke (EDG Dortmund) und zahlreiche Teilnehmerinnen und Teilnehmer arbeiteten in der Diskussion einige zentrale Aspekte heraus:

Der Zusammenhang zwischen Arbeit, Alter(n) und Gesundheit ist mittlerweile gut erforscht. Man weiß, dass Ressourcen (z.B. ergonomische Hilfen bis hin zu Qualifizierungen) die negativen Folgen von Belastungen ab- 
federn können. Als besonders alterskritische Belastungen gelten: Insbesondere hohe physische Anforderungen, die sich bei älteren Beschäftigten stark negativ auswirken. Bei den Maßnahmen zur Förderung alternsgerechter Arbeit existiert ein Gendergap. Physisch stark belastende Tätigkeiten mit hohem Frauenanteil, beispielsweise der Pflegebereich, erhalten zu wenig Unterstützung. Gesundheitliche Einschränkungen sind in diesen Berufen im rentennahen Alter fast der Normalfall. Der Wunsch, möglichst früh in Rente zu gehen, ist immer noch sehr verbreitet und sozial akzeptiert. Gleichwohl entzieht sich der Zusammenhang zwischen Arbeit, Alter und Gesundheit monokausalen Erklärungen und bleibt komplex. Viele Ältere mit gesundheitlichen Einschränkungen arbeiten weiter, und es geht auch bei Älteren gesundheitlich nicht immer nur „bergab“. Die gesundheitliche Situation kann sich im Zeitverlauf wieder verbessern. Dennoch: Es besteht großer Bedarf für Verbesserungen in der Praxis.

Alter(n)sgerechte Arbeitsgestaltung muss früh und systematisch greifen. Von besonderer Bedeutung ist dabei der Blick auf die gesamte Berufsbiografie. Nur so kann Prävention gelingen. Man dürfe nicht erst ansetzen, wenn bereits Einschränkungen vorliegen. Präventive Arbeitsgestaltung stößt jedoch teilweise sogar bei den Beschäftigten auf Widerstand. Beispiel: Aus Präventionsgründen ist ein Tätigkeitswechsel angezeigt, Beschäftigte möchten aber „ihr“ Team nicht verlassen. Wichtigster Punkt für erfolgreiche betriebliche Maßnahmen bleibt jedoch, dass Age-Management von der Unternehmensleitung getragen und aktiv gefördert werden muss.

2. Weiterarbeiten im Rentenalter. Jürgen Deller (Leuphana Universität Lüneburg) und Laura Naegele (Universität Vechta) stellten den Stand des Wissens vor. In der Diskussion mit Jan Oltmanns (Mercedes-Benz AG Bremen) und den Diskussionsteilnehmerinnen und -teilnehmern wurden folgende Befunde besprochen: Entscheidend für die Bereitschaft, im Rentenalter weiterzuarbeiten, sind die Gestaltung und die Qualität der Arbeit. Im Rentenalter sind vorwiegend gesunde, gebildete Menschen in kleineren Betrieben erwerbstätig. Aus gesellschaftlicher Sicht ändert sich der Blick auf erwerbstätige Rentnerinnen und Rentner bereits. Wenn man die Akzeptanz von Menschen im Rentenalter, die einer Berufstätigkeit nachgehen, weiter erhöhen möchte, ist eine gesellschaftliche Diskussion vonnöten.

In den Unterschieden der Erwerbstätigkeit im Rentenalter können sich auch soziale Ungleichheiten ausdrücken. Viele arbeiten nicht in den Bereichen, die ihren Kompetenzen entsprechen, sondern in niedriger qualifizierten Bereichen. Es kann so zu einer Dequalifizierung der Beschäftigten kommen. Vereinbarkeitsproblematiken aufgrund von Care-Tätigkeiten können sich auch im Rentenalter fortsetzen. Zudem ist das Motiv der Frei- 
willigkeit zu diskutieren. Es sind auch diejenigen in den Blick zu nehmen, die aus wirtschaftlichen Gründen arbeiten müssen. Wünschenswert wäre es, wenn die Unternehmen gemeinsam mit ihren Mitarbeiterinnen und Mitarbeitern vor Eintritt des Rentenalters den Übergang vorbereiten und gestalten. Viele Beschäftigte wollen im Rentenalter etwas Neues tun oder andere Kompetenzen einbringen. Hier braucht es Kompetenzentwicklung. Gefragt ist in diesem Zusammenhang eine mitarbeiterorientierte Führung.

3. Soziale Ungleichheit und Gender. Aus dieser Perspektive blickten Martin Brussig (Universität Essen-Duisburg) und Silke Tophoven (Hochschule Düsseldorf) auf die Chancen und Risiken des gesunden Alterns in der Arbeitswelt. Um weitere Aspekte ergänzt wurde dies von Andrea Kreipl-Möller (Deutsche Telekom Technik GmbH) und den Diskussionsteilnehmerinnen und -teilnehmern. Im Mittelpunkt standen die folgenden Erkenntnisse: Derzeit empfinden viele Menschen das hoch gesetzte Rentenalter als versteckte Rentenkürzung - denn sie haben nicht das Gefühl, dass sie gesund bis zum neuen Rentenalter arbeiten können. Tatsächlich verlassen viele Beschäftigte das Erwerbsleben vor dem offiziellen Renteneintrittsalter. Sie müssen also Rentenkürzungen hinnehmen oder die fehlenden Beiträge aus eigener Tasche ausgleichen - was vielen nicht gelingt. Unternehmen sind gefragt, um die Lücke zwischen dem Austritt aus dem Erwerbsleben und dem Renteneintritt zu schließen. Aber auch die Politik muss die Betriebe bei der Verwirklichung einer alters- und alternsgerechten Arbeitsgestaltung unterstützen. Viele Unternehmen haben bislang noch keine ausreichenden Konzepte für ein gesundes Altern in der Erwerbstätigkeit. Natürlich werden Beschäftigte in vielen Unternehmen weiterqualifiziert, es gibt Altersteilzeit oder betriebliches Eingliederungsmanagement, aber weitere, vor allem individualisierte Arbeitsgestaltungskonzepte gibt es kaum. Für viele Unternehmen ist immer noch die Frage offen, was für eine alters- und alternsgerechte Arbeitsgestaltung zu tun ist gerade vor dem Hintergrund, dass es unterschiedliche Beschäftigtengruppen mit unterschiedlichen Bedürfnissen gibt und dass spezifische und individualisierte Arbeitsgestaltungsangebote erforderlich sind.

Die Vereinbarkeit von Arbeit und Familie ist zentral für gesundes Altern in der Erwerbstätigkeit und finanzielles Auskommen im Alter. Insbesondere für Frauen spielt Vereinbarkeit eine zentrale Rolle, um am Arbeitsleben zu partizipieren. Eine gute Vereinbarkeit hat einen positiven Aspekt für die Gesundheit. Auch bei Älteren hat die Verwirklichung der Vereinbarkeit von Arbeit und Leben in Deutschland im Vergleich zu den skandinavischen Ländern noch Nachholbedarf.

4. Lernen und Motivation. Dieses Themenfeld bereiteten Niclas Schaper (Universität Paderborn) und Claudia Kardys (FOM Essen) auf. Corin- 
na Vogt (ING-DiBa AG) brachte die betriebliche Perspektive in die Diskussion ein. Im Mittelpunkt der Diskussion standen die folgenden Erkenntnisse:

Gesundheitsförderliche, alternsgerechte und lernförderliche Arbeitsgestaltung gehören zusammen. Dabei geht es um die Themen Gesundheit, Arbeitsfähigkeit und Prävention. Möglicherweise müssen die Konzepte besser verknüpft und kommuniziert werden. Braucht es neue Konzepte für Lernen und Motivation in Zeiten fortschreitender Digitalisierung? Neue, sich verbreitende Arbeits- und Organisationsformen wie agiles Arbeiten können Lern- und Motivationschancen bieten, aber manche Menschen auch überfordern. Gleichzeitig kann die Digitalisierung für einige Beschäftigte auch zu einer neuen „Taylorisierung“ von Arbeitstätigkeiten führen, was Lernchancen verhindern würde. Es gibt bisher keinen integrierten Ansatz für Lernen und Motivation in der „Arbeitswelt 4.0“.

Das Thema "Gender" ist in Bezug auf Lernen und Arbeitsgestaltung bisher vernachlässigt: Klassische Konzepte (wie lernförderliche Arbeitsgestaltung) wurden im Kontext typischer Männerbranchen (Produktion) entwickelt. Es sollten Konzepte in Bezug auf typische Frauenberufe (Dienstleistung, Care etc.) überprüft und weiterentwickelt werden. Benötigen wir eine geschlechterspezifische Arbeitsgestaltung? Lernen Frauen und Männer anders?

5. Betriebliche und tarifliche Regelungen. Eine Bilanz der Regelungen zu alternsgerechter und altersgerechter Arbeit zogen Peter Guggemos (Hochschule der Bundesagentur für Arbeit) und Kerstin Guhlemann (Sozialforschungsstelle TU Dortmund). Aus der Perspektive der betrieblichen Praxis ergänzte Wolfgang Anlauft (ffw Nürnberg) diesen Blick. Die wichtigsten inhaltlichen Punkte in diesem Themenfeld waren: Tarifverträge und Betriebsvereinbarungen gelten überwiegend in Bereichen wie der Produktion (z.B. Chemieindustrie) und der Großindustrie (z.B. Stahlindustrie), also der Arbeitswelt des männlichen, deutschen Facharbeiters. Gerade in den industriellen Bereichen könnte das Muster des sozialverträglichen Personalabbaus durch Aussteuern der Älteren allerdings schnell wiederaufleben.

Die Weiterentwicklung der Erkenntnisse und ihre Anwendung auf andere, z.T. auch neue Belastungskonstellationen steht auf der Tagesordnung. Die digitale Transformation der Arbeit, aber z.B. auch die Interaktionsarbeit sind hinsichtlich langfristiger Folgen für die Arbeitsfähigkeit bislang kaum erforscht. Es fehlt auch an (Langzeit-) Studien insbesondere zum Erhalt der kognitiven Leistungsfähigkeit in den o.g. Arbeitswelten. Das schnelle Altern junger Beschäftigter in jungen Branchen und Betrieben ist nur ansatzweise erforscht. Mit Blick auf eine erwerbsbiografische 
Orientierung der Forschungen zur Arbeitsfähigkeit verdient die Frage des „berufsbedingten Voralterns“ in jungen Jahren (zum Beispiel in der Gastronomie) mehr Aufmerksamkeit. Es fehlt weiterhin an Institutionen und Strategien, die Erkenntnisse der alter(n)sgerechten Arbeitsgestaltung zuverlässig an KMU sowie Unternehmen ohne betriebliche Interessenvertretung vermitteln.

6. Personalmanagement. Hier arbeiteten Frerich Frerichs (Universität Vechta) und Max Wilckens (Leuphana Universität Lüneburg) den Stand der Erkenntnisse sowie die wichtigsten Erkenntnislücken heraus. Katharina Greiff (Deutsche Bahn AG) sowie die Diskussionsteilnehmerinnen und -teilnehmer ergänzten diese Perspektive. Zentrale Diskussionspunkte waren: Die Auswirkungen der Anhebung der Altersgrenzen (Heraufsetzen des Renteneintrittsalters) auf die Lebenschancen der Beschäftigten werden vielfach unterschätzt. Statt altersbezogene Defizite (die häufig pauschal unterstellt werden) zu bearbeiten, müsste kreativer die längere Erwerbsbiografie gestaltet werden.

Altern ist hochgradig individuell! Alternssensibles Personalmanagement meint das Management der Belegschaften im Betrieb und nimmt damit eine altersübergeordnete Rolle ein. Die kreative Gestaltung der Erwerbsbiografie kann auf dem Konzept der „Lebensphasenorientierung“ aufsetzen. Dieses Konzept muss aber dringend ergänzt werden um integrative Handlungskonzepte. Denn alternsgerechtes Personalmanagement steht im Spannungsfeld von Demografie (konkret: Belegschaftsstrukturen), Generationenzusammenarbeit und Lebensphasenorientierung.

Prävention spielt auf der individuellen Ebene eine immer größere Rolle. Personalmanagement muss also in immer wiederkehrenden Abständen individuelle Perspektiven eröffnen und präventiv wirkende Angebote machen.

Der Erfolg eines präventiven Personalmanagements bemisst sich am Grad der Partizipation der Beschäftigten. Die Qualität eines alternsgerechten Personalmanagements kann (nur) durch sozialpartnerschaftliches Austarieren gesichert werden.

7. Arbeitsgestaltung. Dieses Thema stand im Mittelpunkt der Ausführungen von Thomas Langhoff (FH Niederrhein) und Carolin Eitner (Thyssenkrupp SE). Die wichtigsten Diskussionspunkte waren:

Knowing-Doing-Gap: Es liegen umfangreiche arbeitswissenschaftliche Erkenntnisse zur Gestaltung alterns- und altersgerechter Arbeit vor. Diese werden in der Praxis leider kaum umgesetzt. Zudem hat die Gestaltungskompetenz in den Unternehmen abgenommen - es gibt heute weniger arbeitswissenschaftliche Expertise in den Betrieben als vor 20 Jahren. Wie gelingt es in Zukunft besser, modellhaft erprobte Verbesserungen in den Re- 
gelbetrieb zu überführen? Welche Akteure müssen gewonnen werden, damit Arbeitsgestaltungsmaßnahmen nachhaltig implementiert werden?

Wirtschaftliche Rahmenbedingungen: Die wirtschaftliche Situation des Unternehmens spielt eine große Rolle bei der Umsetzung alternsgerechter Arbeitsgestaltung. Der Kostendruck ist in vielen Unternehmen hoch.

Soziale Rahmenbedingungen: Was bedeutet es für die Beschäftigten, wenn immer weniger Unternehmen tariflich gebunden sind? Führt dies zu einer weiteren Spaltung, sodass bei den tariflich nicht gebundenen Unternehmen nur noch ein Minimalstandard geregelt ist?

Demografieverträge: Demografieverträge werden als sinnvolles Instrument anerkannt, erreichen aufgrund der geringer werdenden Tarifbindung aber immer weniger Arbeitnehmerinnen und Arbeitnehmer. Für die Verbände scheint die tarifliche Regulierung der alternsgerechten Arbeitsgestaltung keine Erfolgsgeschichte zu sein, denn es ist trotz intensivem Bemühen nicht gelungen, einen Verbands- oder Gewerkschaftsvertreterinnen und -vertreter für die Diskussion auf dieser Tagung zu gewinnen. Weitere offene Fragen sind: Inwieweit müssen bei einer präventiven Lebenslaufplanung sowohl die Sozialpartner als auch die Sozialversicherungen stärker als bisher Verantwortung übernehmen? Wie kann hier das „Schnittstellenmanagement" verbessert werden?

8. Podiumsdiskussion zum Abschluss. Die zentrale Frage für Arbeitsgestaltung und Personalpolitik der nächsten Dekade lautet: Wie wird das Age-Management der 2020er-Jahre besser als das der 2010er-Jahre? Darauf gaben Otto Bode (Referatsleiter beim BMBF), Reimund Overhage (Referatsleiter beim BMAS), Anita Tisch (Gruppenleiterin der BAuA), Karl Kuhn und Corinna Vogt (ING) Antworten. Im Mittelpunkt der Diskussion standen folgende Aspekte:

Von den heute Älteren kann nicht umstandslos auf die Älteren geschlossen werden, die aus der heute jungen Generation erwachsen. Das bedeutet, dass weiterhin ein hoher Forschungsbedarf besteht und Erkenntnisse nicht ungeprüft übertragen werden können.

Im betrieblichen Alltag ist ein Suchen und Ausprobieren vonnöten, um herauszufinden, wie und was Älteren und dem Betrieb nützt. Pilotprojekte gerne auch verschränkt mit wissenschaftlicher Evaluation - können gute Ansätze zeigen, die man dann weiterverfolgen kann.

Die sehr gut besuchte Tagung profitierte insbesondere von der Beteiligung von Studentinnen und Studenten der Universitäten Paderborn und Vechta sowie der TU Dortmund. Die Studentinnen und Studenten beteiligten sich im Rahmen eines Gallery Walks mit Postern aktiv an der Tagung und haben auch erheblich zu den lebhaften Diskussionen in den Fishbowlrunden beigetragen. Ein Tagungsband in der Edition Sigma ist in Vorbereitung. 
